\title{
Latent Class Trajectories of Biochemical Parameters and Their Relationship With Risk of Mortality Among Acute Organophosphorus Poisoning Patients
}

\author{
Waqas Ahmed Farooqui ( $\nabla$ waqas.ahmed@duhs.edu.pk) \\ Dow University of Health Sciences \\ Mudassir Uddin \\ University of Karachi \\ Rashid Qadeer \\ Dow University of Health Sciences \\ Kashif Shafique \\ Dow University of Health Sciences
}

\section{Research Article}

Keywords: Latent Class, Organophosphorus poison, Biochemical parameters, Repeated Measures

Posted Date: February 4th, 2021

DOl: https://doi.org/10.21203/rs.3.rs-156166/v1

License: (c) (1) This work is licensed under a Creative Commons Attribution 4.0 International License.

Read Full License 


\section{Abstract}

Objectives: To determine varying biochemical parameters and their relationship with mortality among organophosphorus poisoning patients through a latent class trajectory analysis.

Methods: It was a retrospective cohort study. Patients with organophosphorus poisoning (OP) were admitted to the Intensive Care Unit (ICU) of Ruth Pfau Civil Hospital Karachi from August 2010 to September 2016. A total of 299 OP poisoning patients' data along with demographic and biochemical parameters were retrieved from medical records. The key outcome measure was in-hospital mortality among acute poisoning patients accounting for gender, age, elapse time since poison ingestion, ICU stay, and biochemical parameters including random blood sugar, creatinine, urea, and electrolytes (sodium, chloride, potassium). The trajectories of parameters were formed using longitudinal latent profile analysis. These trajectories and repeated measures were used as independent variables to determine and compare the risk of mortality by Cox-Proportional-Hazards models. A p-value of $<0.05$ was considered statistically significant.

Results: A total of 299 patients' data were included with a mean age of $25.4 \pm 9.7$ years and in-hospital mortality was $13.7 \%(n=41)$. In trajectory analysis, patients with high-declining and normal-increasing creatinine, high-remitting, and normal-increasing urea, high-remitting sodium, trajectories observed the highest mortality i.e. $67 \%(2 / 3), 75 \%(6 / 8), 67 \%(2 / 3), 75 \%(6 / 8)$, and $80 \%(4 / 5)$ respectively compared with other trajectories. On multivariable analysis, patients in high-declining creatinine class were sixteen times [HR:15.7,95\%Cl:3.4-71.6], normal-increasing was fifteen times [HR:15.2,95\%Cl:4.2-54.6] more likely to die compared with those who had normal consistent creatinine levels. Patients in extremely high-remitting urea trajectory were fifteen times [HR:15.4,95\%Cl:3.4-69.7], normal-increasing urea trajectory was four times [HR:3.9,95\% Cl:1.4-11.5] and in high-remitting sodium, the trajectory was six times [HR:5.6,95\%C.I:2.0-15.8] more likely to die compared with those who were in normal-consistent trajectories of urea and sodium respectively.

Conclusion: Using the latent profile approach, biochemical parameters (creatinine, urea, and sodium levels) were significantly associated with increased risk of mortality among OP poisoning patients.

\section{Background}

Acute Poisoning is a global public health challenge [1]. The proportion of suicides due to pesticide selfpoisoning varies in low- and middle-income countries between regions, from $0.9 \%$ in the European region to $48.3 \%$ in the Western Pacific region [2]. Among other poisonings, the prevalence of OP poisoning vary in neighboring countries (7.7\% in Iran [3], 20.7\% in China and India [4, 5]). Among all poisoning cases, the prevalence of OP poisoning was stated closely $46.1 \%$, and due to OP poisoning, $2.7 \%$ mortality reported in a study from our city [6].

Several factors played role in high mortality among acute OP poisoning patients including the age of patients, gender, type amount of poison ingested and its biochemical properties, time since ingestion, any 
pre-existing comorbidities, and access to health services, influence the outcome of OP poisoning patients [7-11]. The prognosis of OP depends on the exposure of toxin, the amount of toxin ingestion, and the physiology of compensation. In our country, it is difficult to judge the amount as patients ingest different brands, which lack the description of concentration of the poisonous substance [6, 12]. In clinical settings, the prognosis of these patients is mainly assessed by a variety of different methods including but not limited to vital status, poisoning scoring systems, and laboratory investigations [5, 13-22]. Biochemical analysis of blood plays an important role in the diagnosis of intoxicated patients since drugs with biochemical substances produce biochemical changes. Studies have linked biochemical parameters (including amylase, lipase, lactate dehydrogenase (LDH), serum immunoglobulins (IgG, $\lg A)$, and creatine phosphokinase (CPK) level) with the severity of OP poisoning but the estimation of these parameters is expensive and most laboratories cannot perform these tests in developing countries.[23, 24] Therefore, there is a need to identify simple and widely useable biochemical parameters in assessing the severity of poisoning as well as the prognosis of OP poisoning patients [20].

Low pseudocholinesterase (PChE), high creatinine $(\mathrm{Cr})$, high sodium $\left(\mathrm{NA}^{+}\right)$, high blood urea nitrogen (BUN), low Glasgow Coma Scale (GCS) scores, and long hospitalization durations have been assessed for their role in OP poisoning patients' prognosis but the findings remain inconclusive among OP poisoning patients $[25,26]$. One of the main reasons might be the conventional approach of using a single baseline measurement of biochemical parameters to predict the mortality of OP poisoning patients. As these biochemical parameters such as level of, random blood sugar, creatinine, blood urea nitrogen, electrolytes, anticholinesterases, red cell distribution width, lactate dehydrogenase, amylase, creatinine kinase, hematocrit, c-reactive protein are dynamic and tend to change substantially over time and also quite dependent on the physiological response of the patient, which vary significantly from patient to patient.

In such a situation, it might not be a suitable method during the follow-up of linking mortality with single measurements at the time of presentation. Because in those studies at baseline only biochemical inquiries were detected, the significant question is, if parameter mean level changes over time, whether that variation leads to some latent classes that are different than the classes made based on a single baseline measurement of the same variable. It might be disposed to to misclassification bias if this single observation method used [1].

Two of the studies reported biochemical investigations among acute OP poisoning patients used repeated measures, one of the studies discussed the use of home perfusion technique and compare blood glucose level and cholinesterase before and after treatment [27], while the other study investigated the predictive value of serum acetylcholinesterase levels measured at five different days and its relationship with different neurological syndromes levels [28].

The classical approach to deal with longitudinal repeated-measures (RM) is RM analysis of variance (ANOVA) but new forms of Structural Equation Modeling (SEM) provides new approaches for repeated measure designs [29]. 
In such a situation, latent growth modeling (LGM) provides a better alternative to observe and estimate growth trajectories overtime for dynamic variables. SEM advances basic longitudinal analysis of data to include latent variable growth over time while modeling both individual and group changes using slopes and intercepts. When these variables are continuous the technique called longitudinal latent class analysis (LLCA) or more specifically longitudinal latent profile analysis (LLPA) [30]. The traditional analysis techniques are analysis of variance, multiple regression, and multilevel models which are variable-centered approaches whereas LCGA is a person-centered approach focused on identifying unobserved subpopulations comprising similar individuals [31]. To the best of our knowledge, there is no previous study that has compared the repeated measures and latent trajectories of biochemical parameters in OP poisoning patients and their relationship with mortality.

Therefore, the present study aimed to analyze the growth trajectory of biochemical parameters among OP poisoning patients and comparing two approaches of biochemical parameters individual response (patterns i.e. LLPA vs RM) and their relationship with mortality using survival analysis.

\section{Methods}

\section{Data source}

All OP poisoning patients admitted during August 2010 to September 2016 at medical ICU of a tertiary care hospital from Karachi-Pakistan i.e. Dr. Ruth K.M. Pfau / Civil Hospital were included in this study. This hospital is one of the largest tertiary care hospitals in the province of Sindh - Pakistan with an annual patient turnover of approximately $>4,000,000$.

\section{Study participants}

A total of 299 OP poisoning patients' older than 13 years of age and whose biochemical parameter data were available were included in this study. Only those patients were included in this study whose at least two to four observations of biochemical parameters with 12 to 24 hours' interval within 0 to 96 hours of admission were available in medical records.

\section{Study design and sample}

This was a retrospective cohort study on OP poisoning patients who was shifted to ICU.

\section{Data collection tool}

A proforma was used to retrieve demographic, clinical characteristics, and biochemical parameters' information.

\section{Ethical consideration}

Ethics approval was obtained from the Institutional Review Board of Dow University of Health Sciences and Board of Advanced Studies and Research (BASR) of the University of Karachi, Pakistan. 


\section{Study variables}

Each patient's data was obtained from medical records.

\section{Independent variables}

Demographics (age, gender), elapse time since poison ingestion and ICU stay. In addition to these variables, biochemical parameters including random blood sugar, creatinine, blood urea nitrogen, sodium electrolyte, chloride electrolyte, potassium electrolyte were also retrieved for an initial period of four days as per completion of medical records. These biochemical parameters were captured overtime during the hospital stay.

\section{Dependent variable}

In-hospital mortality data were recorded to assess the outcome of patients within hospital stay.

\section{Statistical Analysis}

The data were entered and managed using MICROSOFT OFFICE 365 EXCEL. LLPA was utilized for identifying unknown latent classes of individuals with respect to measurements at different time points. It does not model changes over time, but the patterns of measures that span multiple time points [31]. LLPA used in MPLUS version 7.0 software to detect patterns of biochemical parameters. Models were prepared as per guidelines [31, 32]. Our models used first-order polynomials observed linear growth pattern and for each class (two/three/four) whichever best on fit indices to find the maximum latent classes. For every individual, we computed the posterior probabilities and latent class. We choose classes based on each latent class proportion size of at least 5 cases (1.5\%) and best-fitting Log-likelihood, LoMendell-Rubin Test, Bootstrap Likelihood-Ratio Test, Akaike Information Criterion (AIC), Bayesian Information Criterion (BIC), sample size adjusted BIC [33].

Based on the modeled graphical shapes, labels were assigned to the trajectories (Fig. 01). These were based on slope and intercept of mean of individual's pattern. Based on the start of biochemical parameters ranges, we defined labels first part (e.g. low, normal, high, extreme) and on their patterns of repeated measure we identified second part (e.g. consistent, stable, declining, increasing, remitting (up then down or vice versa)). Form of multiple repetition of high and low observations refers to the term remitting during the course of observations i.e. 4 days starting from high to low or vice versa [1].

We computed the repeated measure point biserial correlation used $\mathrm{R}$ version 3.5.1 software.

For inferential analysis and figures used STATA version 15.0 software. To detect differences in age, total ICU stays and baseline biochemical parameters between dead and alive patients, Wilcoxon rank-sum test was applied. Association between gender and biochemical parameters latent classes with mortality were assessed using chi-square and Fisher exact test where appropriate. 
For survival analysis, total ICU stay time was used to define risk time. We computed unadjusted and adjusted hazard ratios (HR) and respective confidence intervals for mortality and the same time compared two approaches using independent variables as assigned trajectory and, in another approach taking independent variables as repeated measures. Cox proportional hazards model was applied. In multivariable analysis, we adjusted for age and approximate time elapsed since ingestion of poison. We evaluated the proportional hazards assumption of Schoenfeld residuals by phtest and power of the Cox Model using Harrell's C concordance statistic; assumptions were identified satisfactory and not violated $[34,35]$. P-values $<0.05$ were considered statistically significant.

\section{Results}

A total of 499 OP poisoning patients (of either gender) were eligible during the six months data collection period from June 2016 to November 2016. Out of the total, 200 patients' records were excluded due to incomplete data, wrong registration, self-reported history of chronic conditions such as hypertension, diabetes mellitus, osteoarthritis, asthma, or pregnant women, less than 3 days ICU stay. A total of 299 patients' data was included for final analysis with a mean \pm standard deviation age of cohort $25.4 \pm 9.7$ years (ranged 13-70 years) with an overall mortality of $13.7 \%(n=41)$. Biochemical parameters (including random blood sugar, creatinine, blood urea nitrogen), age, elapsed time since ingestion of poison were significantly higher among dead as compared to alive individuals, while gender, total ICU stay, and electrolytes were not significantly different between dead and alive individuals. (Table 01) 
Table 01

Descriptive Statistics of Baseline Characteristics with Mortality

\begin{tabular}{|c|c|c|c|}
\hline Characteristics & $\begin{array}{l}\text { Alive } \\
(\mathrm{N}=258,86.3 \%)\end{array}$ & $\begin{array}{l}\text { Dead } \\
(N=41,13.7 \%)\end{array}$ & P-value \\
\hline \multicolumn{4}{|l|}{ Gender } \\
\hline Female & $131(87.3)$ & $19(12.7)$ & \multirow[t]{2}{*}{$0.598^{\sim}$} \\
\hline Male & $127(85.1)$ & $22(14.9)$ & \\
\hline Age (years) & $24.5 \pm 8.1$ & $31.7 \pm 15.0$ & $0.009^{\rrbracket}$ \\
\hline Elapse Time (hours) & $5.6 \pm 3.5$ & $10.1 \pm 3.1$ & $<0.001^{\rrbracket}$ \\
\hline ICU Stay (days) & $5.7(3.8-10.8)$ & $7.5(4.1-10.5)$ & $0.519^{\rrbracket}$ \\
\hline \multicolumn{4}{|l|}{ Biochemical } \\
\hline RBS (mg/dl) & $113.0(100.0-151.0)$ & $143.0(105.0-185.0)$ & $0.018^{\rrbracket}$ \\
\hline $\mathrm{Cr}(\mathrm{mg} / \mathrm{dl})$ & $0.7(0.6-0.9)$ & $0.9(0.7-1.1)$ & $0.009^{\rrbracket}$ \\
\hline BUN (mg/dl) & $10(7-12)$ & $11(9-15)$ & $0.012^{\rrbracket}$ \\
\hline \multicolumn{4}{|l|}{ Electrolytes } \\
\hline Sodium $\left(\mathrm{Na}^{+}\right)(\mathrm{mEq} / \mathrm{L})$ & $141(138-143)$ & $140(137-143)$ & $0.600^{\rrbracket}$ \\
\hline Chloride $\left(\mathrm{Cl}^{-}\right)(\mathrm{mEq} / \mathrm{L})$ & $105(102-108)$ & $104(101-107.5)$ & $0.555^{\rrbracket}$ \\
\hline Potassium $\left(\mathrm{K}^{+}\right)(\mathrm{mEq} / \mathrm{L})$ & $3.9(3.5-4.1)$ & $3.9(3.5-4.1)$ & $0.564^{\rrbracket}$ \\
\hline
\end{tabular}

A repeated measure point bi serial correlation was obtained of biochemical parameters with mortality and highly significant positive correlation was found for creatinine $(r=0.26, p$-value $<0.0001)$ and urea $(r=$ 0.35 , $p$-value $<0.0001)$. While significant but weak correlations were found with mortality for random blood sugar $(r=0.07, p$-value $=0.025)$ and sodium $(r=0.02, p$-value $=0.026) .($ Table 02$)$ 
Table 02

Repeated Measure Correlation of Biochemical Parameters with Mortality

\begin{tabular}{|lll|}
\hline Parameters (Units) & R (P-value) & $95 \%$ C. I \\
\hline Random Blood Sugar $(\mathrm{mg} / \mathrm{dl})$ & $0.07(0.025)$ & $(0.01,0.13)$ \\
\hline Creatinine $(\mathrm{mg} / \mathrm{dl})$ & $0.26(<0.001)$ & $(0.20,0.31)$ \\
\hline Blood Urea Nitrogen $(\mathrm{mg} / \mathrm{dl})$ & $0.35(<0.001)$ & $(0.30,0.40)$ \\
\hline Sodium $\left(\mathrm{Na}^{+}\right)(\mathrm{mEq} / \mathrm{L})$ & $0.02(0.415)$ & $(-0.04,0.08)$ \\
\hline Chloride $\left(\mathrm{Cl}^{-}\right)(\mathrm{mEq} / \mathrm{L})$ & $0.07(0.026)$ & $(0.01,0.13)$ \\
\hline Potassium $\left(\mathrm{K}^{+}\right)(\mathrm{mEq} / \mathrm{L})$ & $002(0.612)$ & $(-0.04,0.08)$ \\
\hline
\end{tabular}

Three distinct trajectories of creatinine (normal-consistent, high-declining and normal-increasing) were identified among 299 OP poisoning patients (Fig. 01), mortality in normal-consistent trajectory was $36 / 292=12.3 \%$, in normal-increasing was $03 / 04=75.0 \%$, while mortality in high-declining trajectory was $02 / 03=66.7 \%)$. (Table 03) 
Table 03

Mortality Comparison of Biochemical Parameters - Latent Classes

\begin{tabular}{|c|c|c|c|c|}
\hline Parameters & $\begin{array}{l}\text { Total } \\
(\mathrm{N}=299)\end{array}$ & $\begin{array}{l}\text { Alive } \\
\mathrm{N}=258 \text { (\%) }\end{array}$ & $\begin{array}{l}\text { Dead } \\
N=41 \text { (\%) }\end{array}$ & P-value \\
\hline \multicolumn{5}{|l|}{ RBS } \\
\hline Normal Stable & 278 & $243(87.4)$ & $35(12.6)$ & \multirow[t]{3}{*}{0.070} \\
\hline Very High Remitting & 07 & $05(71.4)$ & $02(28.6)$ & \\
\hline High Declining & 14 & $10(71.4)$ & $04(28.6)$ & \\
\hline \multicolumn{5}{|l|}{ Creatinine } \\
\hline Normal Consistent & 292 & $256(87.7)$ & $36(12.3)$ & \multirow[t]{3}{*}{0.001} \\
\hline High Declining & 03 & $01(33.3)$ & $02(66.7)$ & \\
\hline Normal Increasing & 04 & $01(25.0)$ & $03(75.0)$ & \\
\hline \multicolumn{5}{|l|}{ Blood Urea Nitrogen } \\
\hline Normal Consistent & 279 & $250(89.6)$ & $29(10.4)$ & \multirow[t]{4}{*}{$<0.001$} \\
\hline High Declining & 09 & $05(55.6)$ & $04(44.4)$ & \\
\hline Extremely High Remitting & 03 & 01 (33.3) & $02(66.7)$ & \\
\hline Normal Increasing & 08 & $02(25.0)$ & $06(75.0)$ & \\
\hline \multicolumn{5}{|l|}{ Sodium } \\
\hline Normal Consistent & 285 & $248(87.0)$ & $37(13.0)$ & \multirow[t]{3}{*}{0.002} \\
\hline High Remitting & 05 & $01(20.0)$ & $04(80.0)$ & \\
\hline Normal Declining & 09 & $9(100.0)$ & $0(0)$ & \\
\hline \multicolumn{5}{|l|}{ Chloride } \\
\hline Normal Consistent & 287 & $250(87.1)$ & $37(12.9)$ & \multirow[t]{3}{*}{0.073} \\
\hline Normal Increasing & 05 & $03(60.0)$ & $02(40.0)$ & \\
\hline High Declining & 07 & 05 (71.4) & $02(28.6)$ & \\
\hline \multicolumn{5}{|l|}{ Potassium } \\
\hline Normal Stable & 272 & $236(86.8)$ & $36(13.2)$ & \multirow[t]{2}{*}{0.392} \\
\hline Normal Increasing & 27 & $22(81.5)$ & $05(18.5)$ & \\
\hline
\end{tabular}


Four trajectories of urea were identified (normal-consistent, high-declining, extreme high remitting, and normal-increasing) (Fig. 01). Patients' mortality in normal-consistent trajectory was $29 / 279=10.4 \%$, in high-declining was $04 / 09=44.4 \%$, in extremely high-remitting was $02 / 03=66.7 \%$, while mortality in normal-increasing trajectory was $06 / 08=75.0 \%$ ). (Table 03)

Three trajectories were identified for sodium electrolyte (normal-consistent, high-remitting, and normal declining) (Fig. 01). Patients' mortality in normal-consistent trajectory was $37 / 285=13.0 \%$, in extremely high-remitting it was $04 / 05=80.0 \%$, while mortality in normal declining trajectory was $0 / 9=0 \%$. (Table 03)

On multivariable analysis after adjusting for age, when modeled repeated measures, creatinine increased the risk of mortality [HR:1.17, 95\% Cl:1.12-1.22, P-value < 0.001]. However, on multivariable analysis after adjusting for age and elapsed time when modeled latent classes, patients with high-declining creatinine were sixteen times more likely [HR:15.7, 95\% Cl: 3.4-71.6, P-value < 0.001] and normal-increasing were fifteen times more likely [HR:15.2, 95\% Cl: 4.2-54.6, P-value < 0.001] to die compared with those who were in normal-consistent trajectory. In the multivariable model, repeated measures analysis, urea showed a significant relation with mortality [HR: 1.01, 95\% Cl: 1.01-1.02, P-value < 0.001]. However, while using latent classes, patients in high-remitting urea trajectory were fifteen times [HR: 15.4, 95\% Cl: 3.4-69.7, Pvalue < 0.001] and in normal-increasing urea trajectory were four times [HR: 3.9, 95\% Cl: 1.4-11.5, P-value $=0.012$ ] more likely to die compared with those who had normal-consistent urea. In multivariable model repeated measures analysis, sodium did not show increased risk of mortality [HR: 1.0, 95\% Cl: 0.9991.001 , P-value $=0.958]$. However, on latent classes, patients in high-remitting sodium trajectory were six times [HR: $5.6,95 \% \mathrm{Cl}: 2.0-15.8$, P-value $=0.001]$ more likely to die compared with those who had normal consistent sodium. (Table 04) 
Table 04

Two Approaches in relationship of mortality with biochemical parameters using Cox Model

\begin{tabular}{|c|c|c|}
\hline \multirow[t]{2}{*}{ Parameters } & Unadjusted & Adjusted \\
\hline & HR (95\% C.I) & $\operatorname{HR}(95 \%$ C.I) \\
\hline \multicolumn{3}{|l|}{ Repeated Measures Approach } \\
\hline Random Blood Sugar (mg/dl) & $1.0(0.999,1.001)$ & $1.0(0.999,1.001)$ \\
\hline Creatinine (mg/dl) & $1.18(1.13,1.22)$ & $1.17(1.12,1.22)$ \\
\hline Blood Urea Nitrogen (mg/dl) & $1.02(1.01,1.02)$ & $1.01(1.01,1.02)$ \\
\hline Electrolytes - Sodium(mEq/L) & $1.0(0.997,1.002)$ & $1.0(0.999,1.001)$ \\
\hline Electrolytes - Chloride(mEq/L) & $1.0(0.996,1.003)$ & $1.0(0.997,1.001)$ \\
\hline Electrolytes - Potassium (mEq/L) & $1.01(0.93,1.11)$ & $1.01(0.98,1.05)$ \\
\hline \multicolumn{3}{|l|}{ Latent Class Approach } \\
\hline \multicolumn{3}{|l|}{ RBS } \\
\hline Normal Stable & 1.0 & 1.0 \\
\hline Very High Remitting & $2.1(0.5,8.6)$ & $2.9(0.7,12.3)$ \\
\hline High Declining & $1.9(0.7,5.4)$ & $1.0(0.3,3.2)$ \\
\hline \multicolumn{3}{|l|}{ Creatinine } \\
\hline Normal Consistent & 1.0 & 1.0 \\
\hline High Declining & $11.6(2.7,49.6)$ & $15.7(3.4,71.6)$ \\
\hline Normal Increasing & $17.5(5.1,60.5)$ & $15.2(4.2,54.6)$ \\
\hline \multicolumn{3}{|l|}{ Blood Urea Nitrogen } \\
\hline Normal Consistent & 1.0 & 1.0 \\
\hline High Declining & $3.5(1.2,10)$ & $2.4(0.8,7.0)$ \\
\hline Extremely High Remitting & $13.1(3.0,55.9)$ & $15.4(3.4,69.7)$ \\
\hline Normal Increasing & $8.2(3.3,20.4)$ & $3.9(1.4,11.5)$ \\
\hline \multicolumn{3}{|l|}{ Electrolyte - Sodium } \\
\hline Normal Consistent & 1.0 & 1.0 \\
\hline High Remitting & $6.2(2.2,17.4)$ & $5.6(2.0,15.8)$ \\
\hline \multicolumn{3}{|l|}{ HR: Hazard Ratio } \\
\hline Adjusted Covariates: (For Repeate & e: Age, For Latent C & Age and Elapse Time \\
\hline
\end{tabular}




\begin{tabular}{|lll|}
\hline Parameters & $\begin{array}{c}\text { Unadjusted } \\
\text { HR }(95 \% \text { C.I })\end{array}$ & $\begin{array}{l}\text { Adjusted } \\
\text { HR(95\% C.I) }\end{array}$ \\
\hline Normal Declining & - & - \\
\hline Electrolyte - Chloride & & \\
\hline Normal Consistent & 1.0 & 1.0 \\
\hline Normal Increasing & $2.0(0.4,11.0)$ & $2.4(0.4,12.8)$ \\
\hline High Declining & $2.3(0.5,9.5)$ & $2.7(0.6,11.9)$ \\
\hline Electrolyte - Potassium & & 1.0 \\
\hline Normal Stable & 1.0 & $0.88(0.46,1.68)$ \\
\hline Normal Increasing & $0.9(0.3,2.3)$ & \\
\hline HR: Hazard Ratio & & \\
\hline Adjusted Covariates: (For Repeated Measure: Age, For Latent Class: Age and Elapse Time $)$
\end{tabular}

\section{Discussion}

Among OP poisoning cases, higher age and longer elapsed time since ingestion were significantly associated with mortality. Patients in high-declining and in normal-increasing creatinine trajectories and those who were in extremely high-remitting and normal-increasing blood urea trajectories, had significantly high mortality compared to patients in normal consistent creatinine trajectory and urea trajectory respectively. Additionally, high-remitting sodium trajectory has significant mortality compared to individuals in normal consistent sodium level trajectory.

The mortality in our study is comparable to previously published papers on OP poisoning from different regions including neighbor countries [5, 6, 13, 26, 36-38], however our study appeared to show higher (13.7\%) mortality compared to another recently published paper $(2.7 \%)$ from National Poisoning Control Centre of our urban city. Low mortality in the previous study was perhaps because the study had only six months records available $(46.1 \%(1174 / 2546))$ for those OP poisoning patients who were managed in medical wards[6] whereas our study spanned over six years patients data recorded from OP poisoning patients admitted in ICU. Longer time elapsed since ingestion of poison and higher age was significant predictors of mortality and findings are quite consistent with previously published studies $[13,26,36]$.

Trajectory analysis showed that two trajectories of creatinine (including high-declining and normal increasing) had a significant relationship with mortality among OP poisoning patients. These results were consistent with previous studies as high creatinine found a significant factor of mortality in multiple Asian studies from 2013 to 2018 including one of our urban cities [13, 17, 22, 26, 39]. Two of Asian studies reported risk of mortality on univariable analysis, patients having high creatinine level founds 
seven to eight times more likely to die [21,40], whereas in our study patients in normal-increasing trajectory were eighteen times and high-declining were twelve times more likely to die compared to the normal consistent creatinine level. This may be due to the mortality within 30 days in these studies whereas in our study it was 20 days.

Trajectory analysis also shown that two trajectories of blood urea nitrogen (including extremely highremitting and normal increasing) were significantly associated with mortality among OP poisoning patients. Our study showed four times and fifteen times increased risk of mortality for normal increasing and extremely high remitting trajectories patients respectively, when compared with normal consistent urea trajectory. These results were somewhat supported by two Asian studies which shown high urea level was associated with mortality [17, 39], and were contrasting with few European and Asian studies which shown urea was not a significant factor of mortality $[13,22,26]$. The LLPA shown in our study that high-remitting trajectory of sodium electrolyte was significantly associated with increased mortality among OP poisoning patients, however previous evidence shown no relationship between sodium and mortality among OP poisoning patients $[13,26]$. The difference of urea and sodium may be due to data collection from the emergency department and a single baseline reading in these studies. The results of random blood sugar from our analysis and previous evidence remains consistent showing no relationship with mortality $[13,26,27]$.

In a comparison of the two techniques repeated measures and latent trajectories, on multivariable analysis, when modeled repeated measure creatinine, and urea level showed a significant relationship with low risk of mortality, while sodium level does not increase the risk of mortality. However, when modeled latent classes patients in high-declining creatinine class were sixteen times, normal-increasing was fifteen times more likely to die compared with those who had normal-consistent creatinine level. Patients in extremely high-remitting urea trajectory was fifteen times, normal-increasing urea trajectory were four times and in high-remitting sodium trajectory were six times more likely to die compared with those who were in normal-consistent trajectories of urea and sodium respectively. This reveals that repeated measure approach is predicted low risk of mortality using creatinine and urea levels while latent class trajectories predicted a high risk of mortality using creatinine, urea, and sodium levels.

Previous studies only included baseline measurements of biochemical parameters and examined their relationship with mortality, while this study was unique in the sense that it accounted for multiple observations of biochemical parameters in the first four days of poisoning and linked them with mortality. Our approach is also closer to real-life scenario, where such OP poisoning patients have varying levels of these parameters, therefore the findings can have more application in clinical settings. This study has examined more accessible, relatively inexpensive biochemical parameters and shown more clinical utility and are much more convenient than expensive laboratory-based markers and may be considered for use in clinical settings for future OP poisoning patients admitted for intensive care. Commonly used scoring systems heavily depends on clinical and laboratory investigational information $[5,17,18,41-45]$, and rely on single measurements usually at the time of admission of patient. LLPA 
used in this study is a person-centered approach where latent classes identify trajectories of patients based on repeated measures of very routine clinical parameters [29].

\section{Strengths and limitations of this study}

- This study included multiple observations of biochemical parameters in the first four days of patients' admission into ICU and determined the relationship of different trajectories of biochemical parameters with mortality among OP poisoning patients.

- To the best of our knowledge, this is the first study that determined the relationship of biochemical parameters with mortality among OP poisoning patients using latent profile approach.

- To the best of our knowledge, this is the first study that compared the two statistical approaches i.e. latent profile research and repeated measures analysis to determine the risk of mortality among OP poisoning patients. The current study had a reasonably larger sample size by including data of several years from ICU of a tertiary care hospital, which provided generalizable results in our context.

- The current study had an estimated amount of poison ingested from medical records, which might not have been very accurate in terms of the actual amount ingested by the patient.

- Our study was only limited to routinely performed biochemical analysis among OP poisoning patients and could not include arterial blood gas such as base deficit and other biomarkers such as anticholinesterases, lactate dehydrogenase because the repeated measures of these markers were not available in medical records for trajectory analysis.

\section{Conclusions}

Our study shows that the latent classes of biochemical parameters, high-declining and normal-increasing trajectories for creatinine, extremely high-remitting and normal-increasing trajectories of blood urea nitrogen, and high-remitting trajectory of sodium electrolyte are highly significant predictors of mortality among acute OP poisoning patients. The latent profile technique appeared to provide better results and prediction compared to conventional repeated measure analysis for such OP poisoning patients.

\section{Abbreviations}

OP: Organophosphorus poisoning; ICU: Intensive Care Unit; LDH: lactate dehydrogenase; CPK: Creatine phosphokinase; PChE: Pseudocholinesterase; Cr: Creatinine; NA+: Sodium; BUN: Blood urea nitrogen; HR: Hazard ratio; RBS: Random Blood Sugar; GCS: Glasgow Coma Scale; RM: Repeated-measures; ANOVA: Analysis of variance; SEM: Structural Equation Modeling; LGM: Latent growth modeling; LLCA: Longitudinal latent class analysis; LLPA: Longitudinal latent profile analysis; IQR: Interquartile range; AIC: Akaike Information Criterion; BIC: Bayesian Information Criterion

\section{Declarations}


Acknowledgements

Authors acknowledge the work of the team. We thank Mariam, Farah, Mahrukh \& Arsalan for helping in data compilation; Dr. Javed Iqbal for proposal for scientific comments \& Dr. Sundus Iftikhar for statistical guidance.

\section{Authors' contributions}

KS and WAF conceived the idea, draft of manuscript and result interpretation, RQ provided clinical perspective, provided access to data, MU helped in literature review, modeling and referencing, WAF performed compilation, transformation, and statistical analysis. All authors read and approved final manuscript.

\section{Funding}

No funding was obtained for this study.

\section{Availability of data and materials}

Data included in the current study are not publicly available to ensure confidentiality of the patients but are available from the corresponding author on reasonable request.

\section{Ethics approval and consent to participate}

Ethics approval was obtained from the Institutional Review Board of Dow University of Health Sciences (DUHS) (Ref No. IRB-560/DUHS/Approval/2015/75 dated 11th Jun 2015) and Board of Advanced Studies and Research (BASR) (BASR/No./02505/Sc. dated 2nd October 2015) of the University of Karachi, Pakistan. All methods were carried out in accordance with relevant guidelines and regulations. The Institutional Review Board of DUHS waived the consent due to the retrospective nature.

\section{Consent for publication}

Not applicable

\section{Competing interests}

The authors declare that they have no competing interests

\section{Authors' information}

${ }^{1}$ Department of Statistics, University of Karachi, Karachi, Pakistan, ${ }^{2}$ School of Public Health, Dow University of Health Sciences, Karachi, Pakistan, ${ }^{3}$ Deparmtent of Medicine, Dr. Ruth K.M. Pfau/Civil Hospital, Dow University of Health Sciences, Karachi, Pakistan, ${ }^{4}$ Institute of Health and Wellbeing, University of Glasgow, Glasgow, United Kingdom. 


\section{References}

1. Farooqui WA, Uddin M, Qadeer R, Shafique K. Trajectories of vital status parameters and risk of mortality among acute organophosphorus poisoning patients - a latent class growth analysis. BMC Public Health. 2020;20(1):1538.

2. Mew EJ, Padmanathan P, Konradsen F, Eddleston M, Chang SS, Phillips MR, et al. The global burden of fatal self-poisoning with pesticides 2006-15: Systematic review. J Affect Disord. 2017;219:93-104.

3. Alizadeh AM, Hassanian-Moghaddam H, Shadnia S, Zamani N, Mehrpour O. Simplified acute physiology score II/acute physiology and chronic health evaluation II and prediction of the mortality and later development of complications in poisoned patients admitted to intensive care unit. Basic Clin Pharmacol Toxicol. 2014;115(3):297-300.

4. Srivastava A, Peshin SS, Kaleekal T, Gupta SK. An epidemiological study of poisoning cases reported to the national poisons information centre, All India Institute of Medical Sciences, New Delhi. Hum Exp Toxicol. 2005;24(6):279-85.

5. Dong N, Liu J, Wang Z, Gao N, Pang L, Xing J. Development of a practical prediction scoring system for severe acute organophosphate poisoning. J Appl Toxicol. 2020.

6. Amir A, Haleem F, Mahesar G, Sattar RA, Qureshi T, Syed JG, et al. Epidemiological, poisoning characteristics and treatment outcomes of patients admitted to the National Poisoning Control Centre at Karachi, Pakistan: a six month analysis. Cureus. 2019;11(11).

7. Hu YH, Chou HL, Lu WH, Huang HH, Yang CC, Yen DH, et al. Features and prognostic factors for elderly with acute poisoning in the emergency department. J Chin Med Assoc. 2010;73(2):78-87.

8. Strøm J, Thisted B, Krantz T, Sørensen MB. Self-poisoning treated in an ICU: drug pattern, acute mortality and short-term survival. Acta Anaesthesiol Scand. 1986;30(2):148-53.

9. Singh O, Javeri Y, Juneja D, Gupta M, Singh G, Dang R. Profile and outcome of patients with acute toxicity admitted in intensive care unit: Experiences from a major corporate hospital in urban India. Indian J Anaesth. 2011;55(4):370-4.

10. Kang EJ, Seok SJ, Lee KH, Gil HW, Yang JO, Lee EY, et al. Factors for determining survival in acute organophosphate poisoning. Korean J Intern Med. 2009;24(4):362-7.

11. Dash SK, Mohanty MK, Mohanty S, Patnaik KK. Organophosphorus poisoning: victim specific analysis of mortality and morbidity. Med Sci Law. 2008;48(3):241-5.

12. Tahir MN, Akbar AH, Naseer R, Khan QO, Khan F, Yaqub I. Suicide and attempted suicide trends in Mianwali, Pakistan: social perspective. East Mediterr Health J. 2014;19 Suppl 3(Suppl 3):S111-4.

13. Ahmed A, Ali L, Shehbaz L, Nasir S, Rizvi SRH, Zaeghum M, et al. Prevalence and characteristics of organophosphate poisoning at a tertiary care centre in Karachi, Pakistan. Pak J Surg. 2016;32(4):269-73.

14. Coskun R, Gundogan K, Sezgin GC, Topaloglu US, Hebbar G, Guven M, et al. A retrospective review of intensive care management of organophosphate insecticide poisoning: Single center experience. Niger J Clin Pract. 2015;18(5):644-50. 
15. Bilgin TE, Camdeviren H, Yapici D, Doruk N, Altunkan AA, Altunkan Z, et al. The comparison of the efficacy of scoring systems in organophosphate poisoning. Toxicol Ind Health. 2005;21(7-8):141-6.

16. Sungurtekin H, Gürses E, Balci C. Evaluation of several clinical scoring tools in organophosphate poisoned patients. Clin Toxicol. 2006;44(2):121-6.

17. Kim YH, Yeo JH, Kang MJ, Lee JH, Cho KW, Hwang S, et al. Performance assessment of the SOFA, APACHE II scoring system, and SAPS II in intensive care unit organophosphate poisoned patients. J Korean Med Sci. 2013;28(12):1822-6.

18. Mohamed S, Hasb Elnabi M, Moussa M, Tawfik H, Adly M. The Accuracy Comparison of Scoring Systems in the Outcome Prediction of Acute Organophosphate Poisoning. Ain Shams J Forensic Med Clin Toxicol. 2019;33(2):8-15.

19. Anormallikleri L. Emergency laboratory abnormalities in suicidal patients with acute organophosphate poisoning. Türk Biyokimya Dergisi [Turkish Journal of Biochemistry-Turk J Biochem]. 2010;35(1):29-34.

20. Dayanand R, Anikethana GV. Liver enzymes for assessment of severity of organophosphorus poisoning. International Journal of Medical and Health Sciences. 2015;4(1):60-3.

21. Kang C, Park IS, Kim DH, Kim SC, Jeong JH, Lee SH, et al. Red cell distribution width as a predictor of mortality in organophosphate insecticide poisoning. The American journal of emergency medicine. 2014;32(7):743-6.

22. Lee YH, Oh YT, Lee WW, Ahn HC, Sohn YD, Ahn JY, et al. The association of alcohol consumption with patient survival after organophosphate poisoning: a multicenter retrospective study. Intern Emerg Med. 2017;12(4):519-26.

23. Sumathi ME, Kumar SH, Shashidhar KN, Takkalaki N. Prognostic significance of various biochemical parameters in acute organophosphorus poisoning. Toxicol Int. 2014;21(2):167-71.

24. Bhattacharyya K, Phaujdar S, Sarkar R, Mullick OS. Serum creatine phosphokinase: a probable marker of severity in organophosphorus poisoning. Toxicol Int. 2011;18(2):117-23.

25. Acikalin A, Disel NR, Matyar S, Sebe A, Kekec Z, Gokel Y, et al. Prognostic Factors Determining Morbidity and Mortality in Organophosphate Poisoning. Pak J Med Sci. 2017;33(3):534-9.

26. Gunduz E, Dursun R, Icer M, Zengin Y, Gullu MN, Durgun HM, et al. Factors affecting mortality in patients with organophosphate poisoning. J Pak Med Assoc. 2015;65(9):967-72.

27. Li Z, Wang G, Zhen G, Zhang Y, Liu J, Liu S. Application of hemoperfusion in severe acute organophosphorus pesticide poisoning. Turk J Med Sci. 2017;47(4):1277-81.

28. Aygun D, Doganay Z, Altintop L, Guven H, Onar M, Deniz T, et al. Serum acetylcholinesterase and prognosis of acute organophosphate poisoning. J Toxicol Clin Toxicol. 2002;40(7):903-10.

29. McArdle JJ. Latent variable modeling of differences and changes with longitudinal data. Annu Rev Psychol. 2009;60:577-605.

30. Vasantha M, Venkatesan P. Structural equation modeling of latent growth curves of weight gain among treated tuberculosis patients. PLoS One. 2014;9(3). 
31. Wang J, Wang X. Structural equation modeling: Applications using Mplus: John Wiley \& Sons; 2019.

32. Muthén B, Muthén BO. Statistical analysis with latent variables: Wiley New York; 2009.

33. Tein JY, Coxe S, Cham H. Statistical Power to Detect the Correct Number of Classes in Latent Profile Analysis. Struct Equ Modeling. 2013;20(4):640-57.

34. Mirza SS, Wolters FJ, Swanson SA, Koudstaal PJ, Hofman A, Tiemeier H, et al. 10-year trajectories of depressive symptoms and risk of dementia: a population-based study. Lancet Psychiatry. 2016;3(7):628-35.

35. Cleves M, Gould W, Gould WW, Gutierrez R, Marchenko Y. An introduction to survival analysis using Stata: Stata press; 2008.

36. Amin DM, Abaza MT, El Azawy DS, Ahmed Al. Morbidity and Mortality Indicators in Acute Organophosphate Poisoning in Zagazig University Hospital, Egypt: Retrospective Study. Occupational Diseases and Environmental Medicine. 2018;6(4):130-40.

37. Mundhe SA, Birajdar SV, Chavan SS. The clinico-demographic study of morbidity and mortality in patients with organophosphate compound poisoning at tertiary care hospital in rural India. Int J Adv Med. 2017;4(3):809.

38. Majidi M, Delirrad M, Banagozar Mohammadi A, Najaf Najafi M, Nekoueifard S, Alizadeh A, et al. Cholinesterase Level in Erythrocyte or Serum: Which is More Predictive of the Clinical Outcome in Patients with Acute Organophosphate Poisoning? IJT. 2018;12(5):23-6.

39. Lee JH, Lee YH, Park YH, Kim YH, Hong CK, Cho KW, et al. The difference in C-reactive protein value between initial and 24 hours follow-up (D-CRP) data as a predictor of mortality in organophosphate poisoned patients. Clin Toxicol. 2013;51(1):29-34.

40. Lee SB, Kang C, Kim DH, Kim T, Lee SH, Jeong JH, et al. Base deficit is a predictor of mortality in organophosphate insecticide poisoning. Hum Exp Toxicol. 2018;37(2):118-24.

41. Sam KG, Kondabolu K, Pati D, Kamath A, Pradeep Kumar G, Rao PG. Poisoning severity score, APACHE II and GCS: effective clinical indices for estimating severity and predicting outcome of acute organophosphorus and carbamate poisoning. J Forensic Leg Med. 2009;16(5):239-47.

42. Wu X, Xie W, Cheng Y, Guan Q. Severity and prognosis of acute organophosphorus pesticide poisoning are indicated by C-reactive protein and copeptin levels and APACHE II score. Exp Ther Med. 2016;11(3):806-10.

43. Senanayake N, de Silva HJ, Karalliedde L. A scale to assess severity in organophosphorus intoxication: POP scale. Hum Exp Toxicol. 1993;12(4):297-9.

44. Davies JO, Eddleston M, Buckley NA. Predicting outcome in acute organophosphorus poisoning with a poison severity score or the Glasgow coma scale. QJM. 2008;101(5):371-9.

45. Persson HE, Sjoberg GK, Haines JA, Pronczuk de Garbino J. Poisoning severity score. Grading of acute poisoning. J Toxicol Clin Toxicol. 1998;36(3):205-13.

\section{Figures}



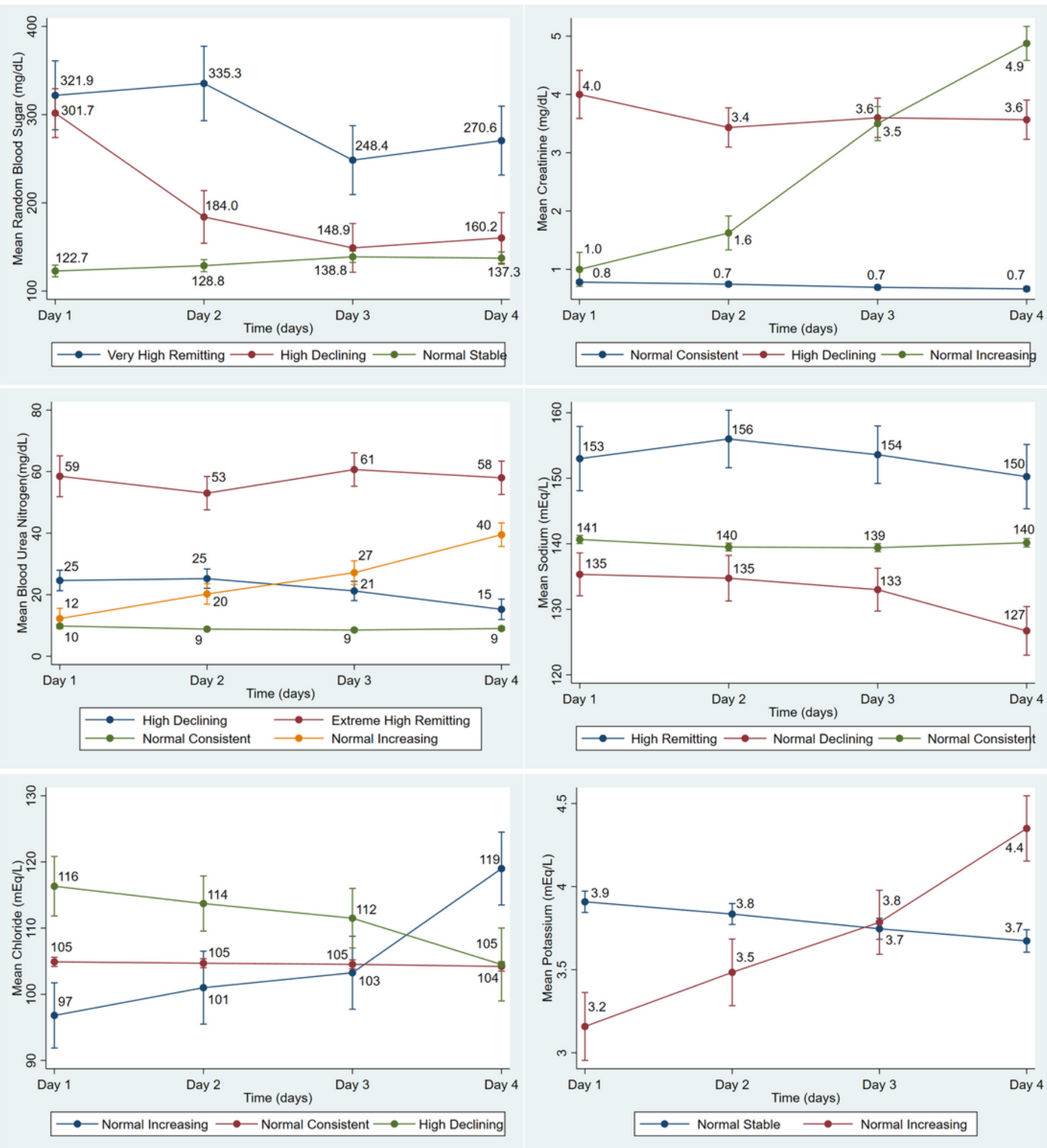

\section{Figure 1}

Trajectories of biochemical parameters from day 1 to 4 